\title{
Correction to: Microdomains in the Cardiovascular System
}

Viacheslav Nikolaev and Manuela Zaccolo

\section{Correction to:}

V. Nikolaev, M. Zaccolo (eds.), Microdomains in the Cardiovascular System, Cardiac and Vascular Biology, DOI 10.1007/978-3-319-54579-0

The original version of this volume was revised as it was originally published unnumbered. The revised version now has been numbered; numbering is done following the order of appearance in the book series Cardiac and Vascular Biology. 